\title{
Parasitisme des tiques (Ixodidae) des bovins à Tiaret, Algérie
}

\author{
A. Boulkaboul ${ }^{1}$
}

\section{Mots-clés}

Bovin - Ixodidae - Metastigmata Tique - Piroplasmose - Algérie.
1. Université Ibn Khaldoun, département des Sciences vétérinaires, Tiaret 14000, Algérie

Adresse personnelle

02, rue du Vieux Palais, Alger 16000, Algérie

\section{Résumé}

Afin de mieux cerner le problème des nuisances et des maladies transmises par les tiques (Ixodidae) aux bovins en Algérie, l'auteur a étudié les caractères saisonniers de leur activité parasitaire dans la région de Tiaret (Algérie). De janvier à décembre, sur un échantillon de 1240 bovins examinés, 368 étaient infestés par des tiques, d'où un taux d'infestation globale d'environ 30 p. 100. La charge parasitaire mensuelle maximale a été de cinq tiques par animal. Parmi 3975 tiques collectées, 13 espèces ont été identifiées, dont six du genre Hyalomma (66 p. 100) et trois du genre Rhipicephalus (31 p. 100). L'espèce numériquement dominante a été $H$. lusitanicum (20 p. 100), alors que $H$. excavatum et $H$. marginatum marginatum ont montré les taux d'infestation des bovins les plus élevés (13 p. 100). Les trois espèces monotropes, reconnues vectrices des piroplasmoses bovines en Algérie, ont représenté 37,4 p. 100 de la population totale des tiques. Parmi ces dernières, Rhipicephalus bursa en a représenté 69 p. 100, Hyalomma detritum 28 p. 100 et Boophilus annulatus 3 p. 100. Le climat semi-aride de la région devait être moins favorable à $B$. annulatus. La présence de $R$. bursa s'est révélée annuelle, alors que celle de $H$. detritum a été limitée à environ quatre mois, en raison d'une diapause hivernale plus prolongée de ses préimagos. La prédominance de $R$. bursa a permis de penser que les babésioses devraient être plus fréquentes dans la région que la théilériose (à Theileria annulata). La faible charge parasitaire observée et les nombreux cas de piroplasmoses signalés chez les bovins supposaient l'existence d'un problème de stabilité enzootique envers ces maladies. A cause d'un risque d'infection potentiel à certaines périodes de l'année, notamment pour les bovins importés, en raison de la prédominance des tiques adultes en saison chaude et des préimagos en saison froide, un traitement acaricide préventif peut être entrepris en mai et en octobre.

\section{INTRODUCTION}

Les tiques (Ixodidae) sont des parasites hématophages communs des animaux domestiques. La nuisance majeure de ces parasites est en rapport avec leur capacité de transmission de certains germes pathogènes pour l'homme et les animaux, notamment les protozoaires sanguins. Ces derniers représentent un véritable fléau pour l'élevage et entraînent de lourdes pertes dans les cheptels atteints.

En Algérie, très peu d'études ont été réalisées à ce sujet. Citons celle conduite à partir de 1921, sur une période de 30 ans, par Sergent et coll. (6), à l'Institut Pasteur d'Algérie. Les auteurs y ont recensé 3875 cas de piroplasmoses chez les bovins (de 1924 à 1945 ) et ont révélé l'implication de trois vecteurs : Boophilus 
annulatus, Rhipicephalus bursa pour les babésioses et Hyalomma detritum pour la theilériose. Ces trois espèces sont toutes du type monotrope : leurs différents stades parasitent un même type d'hôte, en l'occurrence les bovins.

Sur la base des données de cette étude, l'auteur s'est intéressé, en particulier, à rechercher l'incidence du parasitisme par ces trois espèces sur les bovins dans la région de Tiaret. Ce travail a été axé sur deux points : l'identification des espèces de tiques inféodées aux bovins de la région et l'étude de l'évolution saisonnière de leur parasitisme, ainsi que son importance.

La connaissance des tiques et de leur biologie est primordiale pour l'étude de l'épidémiologie des maladies transmises qui apparaissent pendant la période d'activité des vecteurs et aussi dans un cadre de prophylaxie, celle-ci devant être effectuée au moment le plus opportun, avec le coût le plus faible.

\section{MATERIEL ET METHODES}

L'étude a concerné des bovins de la région de Tiaret, sans distinction de race, d'âge ou de sexe.

\section{Données géo-climatiques de la région}

La wilaya (district) de Tiaret est située à $300 \mathrm{~km}$ au sud-ouest d'Alger (figure 1). Son relief varie avec des altitudes comprises entre 800 et $1200 \mathrm{~m}$. C'est une zone agropastorale, à climat de type méditerranéen, continental. Elle se situe entre les isohyètes 250 et $500 \mathrm{~mm}$. La moyenne thermique maximale $\left(26^{\circ} \mathrm{C}\right)$ est enregistrée au mois d'août et la moyenne minimale $\left(6^{\circ} \mathrm{C}\right)$, au mois de janvier. On y relève l'importance de la saison chaude et sèche qui peut s'étendre sur six mois (de mai jusqu'à octobre).

\section{Technique de récolte des tiques}

Les tiques ont été récoltées de façon hebdomadaire : dans les marchés du bétail de la wilaya de Tiaret, dans trois localités (Tiaret, Sougueur, Frenda), et dans des fermes privées réparties sur toute la wilaya (figure 2). Les fermes privées ont été choisies au hasard, bien que concentrées dans la partie septentrionale de la wilaya, les zones situées plus au sud étant appauvries en élevage bovin. De même, au niveau des marchés, les bovins ont été choisis au hasard.

Les animaux ont été minutieusement contrôlés et complètement détiqués à la main. Dans certains cas, les larves, à cause de leur très faible taille, n'ont pas été prélevées en totalité. La majorité des échantillons ont ensuite été conservés dans des flacons contenant de l'éthanol à $70^{\circ}$, mais une partie a été gardée en vie, pour étude en laboratoire.

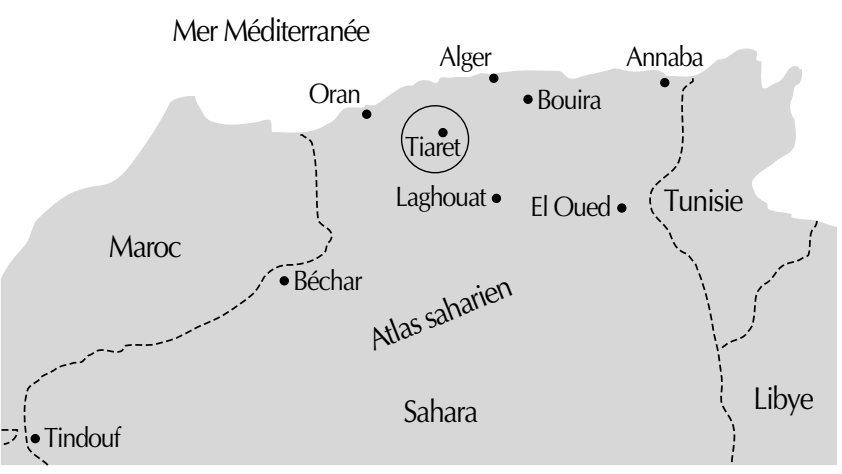

Figure 1 : carte d'Algérie avec la situation de la wilaya (district) de Tiaret.

\section{Identification des tiques}

L'identification des stades adultes a été réalisée au laboratoire à l'aide d'une loupe binoculaire. La diagnose des genres a été basée sur les caractères morphologiques de certaines parties du corps de la tique (rostre, yeux, festons). La diagnose des espèces a été basée sur certains détails morphologiques (ponctuation du scutum, coloration des pattes, forme des stigmates, caractères des sillons, des festons et des yeux). En ce qui concernait les stades préimaginaux, plus difficiles à identifier, l'auteur a procédé de la façon suivante : les tiques vivantes ont été placées à l'étuve à haute température $\left(30^{\circ} \mathrm{C}\right)$ et 70 p. $100 \mathrm{HR}$, afin d'obtenir après quelques jours les adultes. Ce qui a permis de confirmer la diagnose faite au début par l'observation du rostre des préimagos, qui présentait un aspect différent entre les espèces monotropes.

\section{Appréciation du parasitisme}

Les critères utilisés pour l'appréciation du parasitisme ont été, d'une part, le taux d'infestation des bovins par les tiques (p. 100) : - le taux d'infestation globale appliqué à l'année (nombre de bovins infestés au cours de l'année/nombre de bovins examinés au cours de l'année) x 100 ;

- le taux d'infestation mensuelle appliqué au mois (nombre de bovins infestés au cours du mois/nombre de bovins examinés au cours du mois) x 100.

Ce taux a été appliqué à l'ensemble des tiques ou à une espèce donnée (indiquant sa prévalence).

D'autre part, les critères utilisés ont été la charge parasitaire des tiques (nombre/animal). Dans ce dernier cas, on pouvait distinguer :

- la charge parasitaire globale (nombre total de tiques récoltées/nombre total de bovins examinés) ;

- la charge parasitaire individuelle (nombre de tiques récoltées sur un animal à un moment donné) ;

- la charge parasitaire mensuelle (nombre de tiques récoltées pendant le mois/nombre de bovins examinés pendant le mois).

La charge parasitaire pouvait concerner l'ensemble des tiques ou une espèce donnée. De même, le taux d'infestation et la charge parasitaire pouvaient être appliqués, dans le cas des espèces monotropes, aux adultes et aux préimagos.

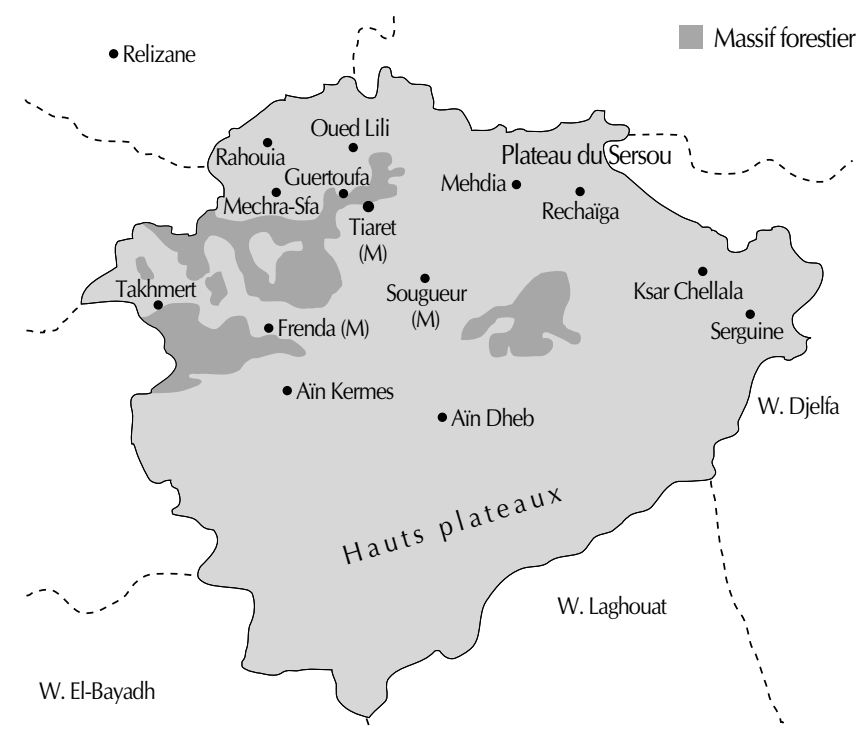

Figure 2 : la wilaya (district) de Tiaret, avec les principales localités concernées par les collectes de tiques. $(M)=$ marchés aux bestiaux hebdomadaires. 


\section{RESULTATS}

\section{Données générales du parasitisme}

L'effectif total des bovins contrôlés a été de 1240 têtes. L'effectif des bovins infestés a été de 368 têtes, d'où un taux global d'infestation de $29,6 \pm 2,5$ p. 100. L'effectif total des tiques récoltées a été de 3975 , dont 82,9 p. 100 adultes et 17,1 p. 100 préimagos. Pour ces derniers, le chiffre a été biaisé, car les larves n'ont pas été récoltées en totalité dans certains cas.

D'après la figure 3 , le taux d'infestation des bovins, variable au cours de l'année, a atteint un maximum en avril (48,2 p. 100) et a montré une plus faible augmentation en octobre. Mais c'est au mois de juin que la charge parasitaire moyenne a été la plus élevée individuelle de 72 tiques par animal.

\section{Espèces de tiques identifiées *}

Le dénombrement des tiques a permis d'identifier 13 espèces, dans six genres (tableau I). La majorité des tiques appartenaient aux genres Hyalomma (66 p. 100) et Rhipicephalus (31 p. 100).

Les espèces de type monotrope (H. detritum, $R$. bursa, Boophilus annulatus), dont tous les stades peuvent être rencontrés sur les bovins, ont été numériquement minoritaires (37,4 p. 100) par rapport aux autres espèces. Les espèces numériquement dominantes ont été $H$. lusitanicum et $H$. marginatum marginatum, représentant respectivement 20 et 17,8 p. 100 des tiques récoltées.

\section{Période d'activité des tiques}

Pour la majorité des espèces, la présence sur les bovins a été saisonnière. Celle-ci est résumée dans le tableau II. On note une succession des espèces et des stades évolutifs au cours de l'année.

* L’identification a été confirmée par le docteur P.C. Morel (†), Iemvt, MaisonsAlfort, France (cinq tiques par animal), avec une charge parasitaire maximale

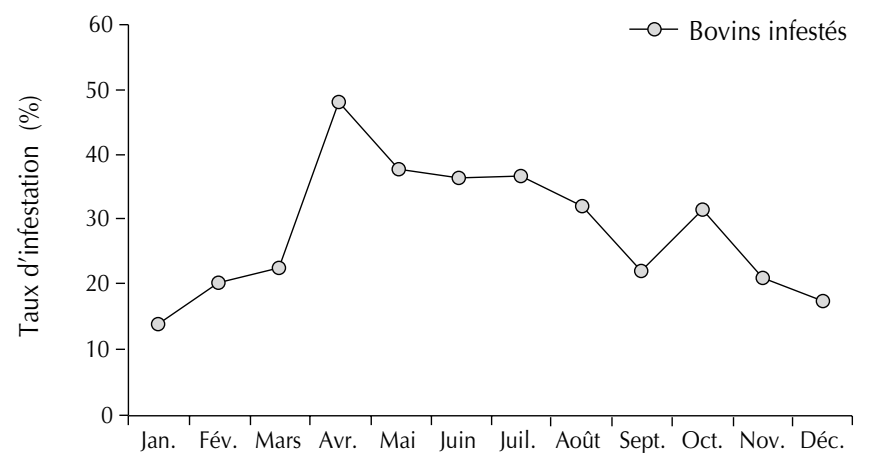

Figure 3 : évolution du taux d'infestation des bovins par les tiques au cours de l'année.

Pendant la saison chaude, c'étaient des espèces thermophiles, telles celles des genres Hyalomma et Rhipicephalus, qui ont été trouvées sur les bovins. Les autres espèces (Haemaphysalis punctata, Ixodes ricinus, Dermacentor marginatus) n'ont été récoltées qu'à partir de l'automne. B. annulatus a été recueilli uniquement en septembre. Les préimagos des deux autres espèces monotropes l'ont été pendant l'automne (H. detritum) et l'hiver (R. bursa).

\section{Association d'espèces}

Plusieurs espèces peuvent parasiter le même animal. L'association minimale de deux espèces a été observée au mois de janvier et l'association maximale (cinq espèces) au mois de juin. Ceci conditionne bien sûr la charge parasitaire d'un animal à un moment donné.

\section{Caractères de l'activité parasitaire des espèces}

La description a été limitée aux espèces les plus dominantes, les autres espèces ayant eu un taux d'infestation inférieur à 1 p. 100.

\section{Tableau I}

Effectifs et proportions relatives des espèces de tiques récoltées

\begin{tabular}{llrc} 
Genres & Espèces & Effectif & $\%$ \\
\hline Hyalomma & lusitanicum & 796 & $20,02 \pm 1$ \\
& excavatum & 669 & $16,83 \pm 1$ \\
& marginatum & 707 & $17,79 \pm 1$ \\
& marginatum & & \\
& detritum & 417 & $10,49 \pm 0,9$ \\
& impeltatum & 37 & $0,93 \pm 0,3$ \\
& marginatum rufipes & 3 & $0,08 \pm 1$ \\
Rhipicephalus & bursa & 1027 & $25,84 \pm 1$ \\
& turanicus & 185 & $4,65 \pm 0,6$ \\
Haemaphysalis & punctata & 12 & $0,30 \pm 0,2$ \\
Boophilus & annulatus & 47 & $1,18 \pm 0,4$ \\
Dermacentor & marginatus & 45 & $1,13 \pm 0,3$ \\
Ixodes & ricinus & 27 & $0,68 \pm 0,3$ \\
Total & & 3 & $0,08 \pm 0,09$ \\
& & 3975 & 100
\end{tabular}

Intervalle de confiance à $5 \%$

\section{Tableau II}

Périodes d'activité parasitaire des espèces de tiques dans la région de Tiaret

\begin{tabular}{|c|c|c|c|}
\hline Printemps & Eté & Automne & Hiver \\
\hline \multicolumn{4}{|c|}{ Hyalomma lusitanicum, $H$. excavatum } \\
\hline \multicolumn{4}{|c|}{ H. marginatum marginatum } \\
\hline \multicolumn{4}{|c|}{$\begin{array}{l}\text { Rhipicephalus turanicus, } \\
\text { R. sanguineus }\end{array}$} \\
\hline \multicolumn{2}{|c|}{ R. bursa (adultes) } & \multicolumn{2}{|c|}{$\begin{array}{l}\text { R. bursa (préimagos), } \\
\text { Haemaphysalis punctata }\end{array}$} \\
\hline & $\begin{array}{c}\text { Hyalomma } \\
\text { detritum } \\
\text { (adultes), } \\
\text { H. impeltatum, } \\
\text { Boophilus } \\
\text { annulatus } \\
\text { (adultes + } \\
\text { préimagos) }\end{array}$ & $\begin{array}{l}\text { H. detritum } \\
\text { (préimagos) }\end{array}$ & \\
\hline $\begin{array}{l}\text { Dermacentor } \\
\text { marginatus }\end{array}$ & & & $\begin{array}{l}\text { Dermacentor } \\
\text { marginatus, } \\
\text { Ixodes ricinus }\end{array}$ \\
\hline
\end{tabular}


D'après la figure 4, sur la base du taux d'infestation, trois espèces ont eu une présence dominante sur les bovins : $H$. excavatum, $H$. m. marginatum (environ 13 p. 100) et $R$. bursa (11 p. 100). Les variations mensuelles du taux d'infestation des espèces ditropes et monotropes dominantes sont indiquées respectivement par les figures 5 et 6 .

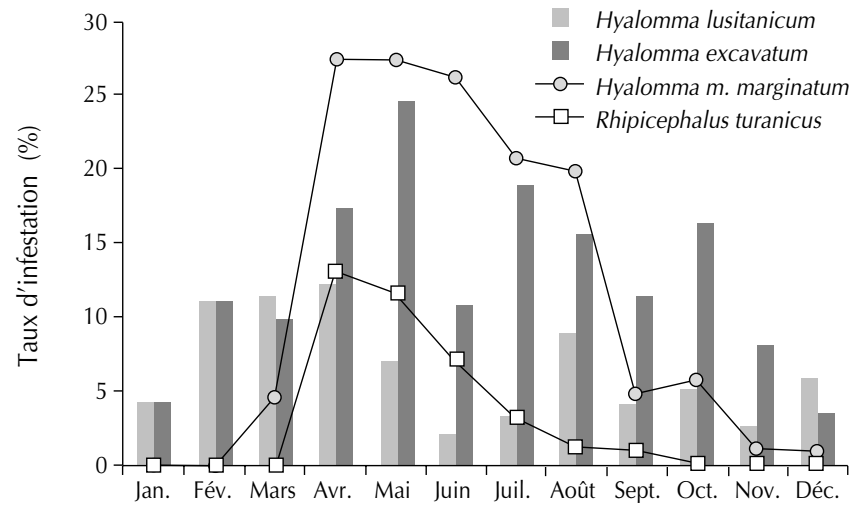

Figure 5 : évolution du taux d'infestation bovine par les espèces ditropes dominantes au cours de l'année.

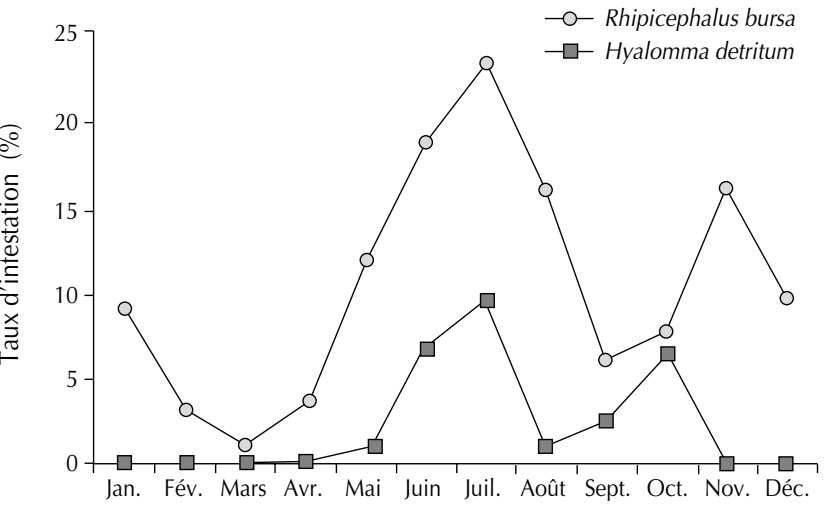

Figure 6 : évolution du taux d'infestation bovine par les espèces monotropes dominantes au cours de l'année.
La charge parasitaire globale (3 975 tiques/1 240 bovins), relative à toute la région, qui était théorique, a été d'environ trois tiques par animal. Cependant, à cause d'une variation entre les espèces, le tableau III montre les charges individuelles maximales réellement observées.

\section{Tableau III}

Charges parasitaires individuelles maximales des espèces de tiques dominantes

\begin{tabular}{lcc} 
Espèces & $\begin{array}{c}\text { Charge } \\
\text { individuelle } \\
\text { maximale * }\end{array}$ & $\begin{array}{c}\text { Mois } \\
\text { de } \\
\text { I'observation }\end{array}$ \\
\hline Hyalomma lusitanicum & 65 & Avril \\
H. marginatum marginatum & 41 & Mai \\
Rhipicephalus turanicus & 22 & Juin \\
H. excavatum & 38 & juillet \\
R. turanicus & 22 & Juin \\
H. detritum (adultes) & 56 & Juillet \\
H. detritum (préimagos) & 44 & Septembre \\
R. bursa (adultes) & 50 & Juin \\
R. bursa (préimagos) & 36 & Novembre
\end{tabular}

* Nombre de tiques par animal

\section{Espèces ditropes}

H. excavatum a été présente sur les bovins pendant toute l'année. Cette espèce a été de loin la plus largement distribuée dans la région. Elle a été rencontrée dans le même biotope que $H$. m. marginatum.

H. m. marginatum a été trouvée sur les bovins depuis mars jusqu'à décembre. L'espèce a été rencontrée dans les biotopes ouverts (prairies et zones de cultures domestiques).

H. lusitanicum a eu une activité annuelle. C'est l'espèce qui a réalisé la plus forte charge parasitaire sur les bovins. Elle a été surtout récoltée dans les zones boisées et buissonneuses de la région.

Rhipicephalus turanicus a eu une activité saisonnière relativement courte, s'étendant d'avril à août. La distribution de l'espèce dans la région a été moins importante que celle de R. bursa (figure 5).

\section{Espèces monotropes}

$R$. bursa a été présente toute l'année, mais avec un caractère saisonnier, partagé entre les adultes (saison chaude) et les préimagos (saison froide). Un seul animal a porté des adultes et des préimagos en même temps. L'espèce a été largement distribuée dans la région, depuis le nord jusqu'à l'extrême sud.

H. detritum a eu une activité saisonnière nette, avec deux pics, partagés entre adultes et préimagos. Contrairement aux résultats de Yousfi-Monod et Aeschlimann (8), dont l'étude a englobé toute l'Oranie, ils devaient hiberner plus tôt : ils n'ont pas été trouvés sur les animaux en novembre et décembre.

B. annulatus, l'espèce principale intervenant dans la transmission des babésioses bovines (6), a été quasiment absente de Tiaret. Sur 1240 bovins examinés, seuls deux ont été parasités par cette espèce. Les deux bovins provenaient d'une même localité, à climat plus humide, située à la limite nord de la région (Rahouia). L'effectif de tiques récolté se composait de 39 adultes et 6 préimagos (figure 6). 


\section{DISCUSSION}

L'étude a tenté de révéler l'importance pathologique potentielle des différentes espèces récoltées sur les bovins. Cette importance est liée à la fois à l'effectif de l'espèce de la tique, au taux d'infestation des bovins, à la durée d'activité sur l'année et à son rôle éventuel dans la transmission d'agents pathogènes aux bovins, dont les piroplasmoses. Par ailleurs, la charge parasitaire conditionne la situation immunitaire des animaux envers ces pathologies, dite « état épidémiologique » (4).

Du point de vue de l'affinité pour les bovins, ont été distinguées des tiques pratiquement ditropes (I. ricinus et Hm. punctata incluses) et des tiques monotropes. L'activité parasitaire des tiques récoltées a été saisonnière et deux groupes ont été différenciés : un groupe actif en saison chaude (espèces thermophiles) et un autre actif en saison froide (espèces mésophiles). Pour les espèces monotropes, les adultes ont prédominé au cours de la saison chaude et les préimagos, plus sensibles à la dessiccation, ont infesté les bovins au cours de l'automne et de l'hiver.

Le taux global d'infestation des bovins, de l'ordre de 30 p. 100, a semblé assez faible, mais sa signification est devenue plus importante lorsque le taux a été considéré au moment de l'activité parasitaire la plus intense. Ainsi, entre avril et juin, le taux d'infestation a atteint 40,4 p. 100 des animaux examinés au cours de ce mois, et le nombre de tiques a représenté alors 46,6 p. 100 de la population totale des tiques récoltées.

La faiblesse du taux global d'infestation devait être la conséquence de la variation saisonnière de l'activité parasitaire des tiques, ainsi que de la concentration des espèces dans des microclimats différents les un des autres (cas de H. lusitanicum).

La charge parasitaire a commencé à grimper au début du printemps. Son augmentation a notamment été liée à l'apparition successive des espèces, entraînant une infestation multiple des bovins, ce qui a expliqué la charge parasitaire individuelle maximale de 72 tiques/animal, observée au mois de juin (cinq espèces sur le même animal). Cependant, la charge mensuelle maximale a été basse (5 tiques/animal), si l'on considère que les bovins peuvent être infestés par des centaines de tiques. En général, dans la région étudiée, les traitements acaricides ne sont pas courants, du fait de la cherté relative de certains produits et de l'ignorance des éleveurs des effets nuisibles représentés par les tiques. Quand ils sont utilisés, les traitements sont réalisés entre juin et juillet, et surtout sur des bovins importés. Cette région appartient à la zones des hauts-plateaux, comportant essentiellement des zones dégagées, pauvres en végétation. La plupart du temps, les animaux parcourent de larges distances pour se nourrir, sans rester suffisamment longtemps au même endroit. A cela s'ajoute l'état immunitaire des animaux qui devait expliquer la faible charge parasitaire observée.

Les espèces numériquement dominantes ont été $H$. lusitanicum (20 p. 100) et $H$. m. marginatum (16,8 p. 100). Elles ne semblent pas être impliquées naturellement dans la transmission de piroplasmoses. Cependant, $H$. m. marginatum étant de taille assez grande entraîne des lésions inflammatoires notables au point de piqûre, notamment au niveau de la mamelle, et peut être à l'origine d'une anémie importante, une tique femelle pouvant prélever $10 \mathrm{ml}$ de sang sur l'animal (2).

Le fait que la charge parasitaire ait été la plus élevée chez $H$. lusitanicum devait être en relation avec son biotope. Ce dernier, caractérisé par une végétation dense, doit offrir une bonne protection aux tiques et empêche, par sa structure, le déplacement rapide des bovins, facilitant ainsi leur infestation. Mais le nombre d'animaux parasités par cette espèce n'a pas été important : 6,4 p. 100 des animaux examinés.

La rareté de $B$. annulatus (taux d'infestation des bovins inférieur à 1 p. 100), vecteur des babésioses bovines en Algérie (6), devait être principalement lié au climat très sec de la région étudiée, cette espèce de type mésophile ayant une affinité pour des zones tempérées humides. Elle doit avoir un rôle pathogène assez réduit dans la région.

$H$. detritum est reconnu vecteur naturel de Theileria annulata en Algérie (6). Cette espèce a été relativement peu présente à Tiaret, avec un taux d'infestation des bovins de 3 p. 100 seulement. La maladie peut être inoculée aux animaux par une transmission transtadiale de Theileria $(1,7)$. On peut supposer qu'elle ne peut être observée sur les bovins de la région qu'entre juin et août.

En revanche, le taux d'infestation des bovins supérieur (11 p. 100) pour $R$. bursa, reconnu vecteur de piroplasmoses bovines en Algérie (6), ainsi que sa présence quasiment annuelle partagée entre adultes et préimagos devaient en faire l'espèce la plus importante dans la région. Son activité plus durable rend ainsi l'apparition des cas de babésioses possible au cours de toute l'année, plus particulièrement entre le printemps et l'automne, sachant que les agents de ces maladies peuvent être inoculés aux bovins par la larve, la nymphe et la femelle dès les premiers jours du repas sanguin $(3,6)$.

Comparativement, selon l'étude de Sergent et coll. (6), les cas de piroplasmoses bovines s'observent sur toute l'année en Algérie, mais la majorité $(80$ p. 100) sont concentrés entre juin et septembre, avec une intervention forte de $B$. annulatus. Dans une autre étude, effectuée au nord-ouest du pays, ce sont en particulier les cas de theilériose qui prédominent, coïncidant avec une fréquence élevée de $H$. detritum (8). L'élevage n'ayant pas changé en Algérie (traditionnel, avec importations ponctuelles de races bovines européennes), on peut estimer que le facteur le plus important dans les variations du parasitisme est d'ordre géo-climatique, les régions de l'est du pays étant plus humides et plus fournies en végétation que les régions de l'ouest.

La lutte contre les tiques doit tenir compte de l'état de prémunition des animaux. Il est logiquement plus fragile pour les sujets importés que pour les sujets autochtones. Sur la base des observations de terrain de cette étude, de nombreux bovins porteurs de tiques étaient fortement amaigris et certains ont succombé à des piroplasmoses malgré les traitements. Le cheptel est-il dans une situation d'instabilité enzootique (4) ? Le nombre de tiques nécessaire au maintien de l'état de prémunition n'étant pas connu, la réponse exige des études plus approfondies à ce sujet.

Cependant l'utilisation d'acaricides semble indispensable à certaines périodes de l'année. La charge parasitaire et le taux d'infestation ont été à leur maximum en été pour les adultes et en automne pour les préimagos. Les traitements peuvent donc être utilisés chez les animaux les plus infestés, en mai et en octobre, afin de diminuer l'incidence des piroplasmoses qui sont fréquemment signalées dans la région au cours de la saison chaude.

\section{- CONCLUSION}

Pour la région étudiée, le présent travail fait apparaître les points suivants : des taux d'infestation bovine et de charge parasitaire faibles (par rapport à des régions de l'est du pays) ; une influence des conditions géo-climatiques sur la répartition des espèces, avec une prédominance d'espèces thermophiles (Hyalomma spp., Rhipicephalus spp.), et une relative faible présence des espèces monotropes, notamment $H$. detritum et $B$. annulatus ; une dominance numérique de trois espèces $(H$. lusitanicum, $H$. excavatum et 
H. m. marginatum), sur lesquelles il faudra approfondir les investigations concernant l'incidence pathologique directe et indirecte ; une dominance de $R$. bursa parmi les espèces monotropes, avec une répercussion probable sur l'apparition des cas de babésioses bovines dans la région ; et un niveau d'activité maximal des stades adultes en été et celui des stades préimaginaux en automne, conditionnant le calendrier des traitements acaricides, mais qui ne doit pas être appliqué de façon systématique. En effet, il est recommandé d'approfondir les études sur la question de l'état de prémunition des bovins, afin de pouvoir déterminer de façon précise les charges parasitaires nécessaires à son maintien. Enfin, il est nécessaire de conforter la possible prédominance des babésioses par rapport à la theilériose par des études épidémiologiques sur ces pathologies elles-mêmes.

\section{Remerciements}

Ce document est dédié à la mémoire du docteur P.C. Morel. J'adresse mes sincères remerciements, pour leur précieuse collaboration, au professeur Phan Trong Cung (université de l'agriculture, Hanoï, Vietnam), ainsi qu'au docteur K. Gadomska (W. Stefanski Institut of Parasitology of Polish Academy of Sciences, Pologne).

\section{Summary}

Boulkaboul A. Parasitism of Cattle Ticks (Ixodidae) in Tiaret, Algeria

In order to better understand the problem of damages and diseases transmitted by ticks (Ixodidae) to cattle in Algeria, the author studied the seasonal features of their parasitic activity in the region of Tiaret (Algeria). From January to December, in a sample of 1240 cattle examined, 368 were infested by ticks, with a global cattle infestation rate of about $30 \%$. The maximum monthly parasitic burden was five ticks per animal. Among 3975 ticks collected, 13 species were identified, with six species of the genus Hyalomma (66\%) and three of that of Rhipicephalus (31\%). The numerically dominant species was $H$. lusitanicum $(20 \%)$, whereas $H$. excavatum and $H$. marginatum marginatum had the highest cattle infestation rates $(13 \%)$. The three monotropic species, recognized as vectors of bovine piroplasmosis in Algeria, represented 37.4\% of the total tick population. Among these, Rhipicephalus bursa represented $69 \%$, Hyalomma detritum 28\%, and Boophilus annulatus $3 \%$. The semiarid climate of the region must have been less favorable to $B$. annulatus. The presence of $R$. bursa was shown to be all year round, whereas that of $H$. detritum was limited to about four months, because of a more prolonged wintry diapause of its preimagos. $R$. bursa predominance indicated that babesioses must have occurred more frequently in the region than theileriosis (caused by Theileria annulata). The low tick burden observed and the numerous cases of piroplasmoses reported in cattle underlied the presence of an enzootic stability problem towards these diseases. Because of a potential infection risk during some periods of the year, notably in imported animal breeds, owing to adult tick predominance in the hot season and preimagos in the cold season, a preventive acaricide treatment can be applied in May and October.

Key words: Cattle - Ixodidae - Metastigmata - Tick - Piroplasmosis - Algeria.

\section{BIBLIOGRAPHIE}

1. BHATACHARYULLU Y., CHAUDRI R.D., GILL B.S., 1975. Transtadial transmission of $T$. annulata through common ixodid ticks infesting Indian cattle. Parasitology, 71: 1-7.

2. KAUFMANN J., 1996. Parasitic infections of domestic animals: a diagnostic manual. Basel, Switzerland, Birkhäuser Verlag.

3. MEHLHORN H., SCHEIN E., 1984. The piroplasms: life-cycle and sexual stage. Adv. Parasitol., 23: 37-103.

4. MOREL P.C., 1981. In : Précis de parasitologie vétérinaire tropicale. Maisons-Alfort, France, lemvt, p. 475-509, 593-623.

5. ROUINA A.D., 1981. Etude clinique de la theilériose bovine sur 237 cas en Algérie. Thèse vétérinaire, OPU, Alger, Algérie, 60 p.

6. SERGENT E., DONATIEN A.L., PARROT L.M., LESTOQUARD F., PLANTUREU E., 1945. Etude sur les piroplasmoses bovines. Alger, Algérie, Institut Pasteur, 770 p.

7. SERGENT ET COLL., 1936. Cycle évolutif de Theileria dispar du bœuf chez la tique Hyalomma mauritanicum. Arch. Inst. Pasteur Algérie, 14 : 259-294.

8. YOUSFI-MONOD R., AESCHLIMANN A., 1986. Recherches sur les tiques (Ixodidae) parasites des bovidés dans I'Ouest algérien. Ann. Parasitol. Hum. comp., 61 : 341-358.

Accepté le 28.05.2004

\section{Resumen}

Boulkaboul A. Parasitismo de las garrapatas (Ixodidae) de los bovinos en Tiaret, Argelia

Con el fin de cernir mejor el problema de la nocividad y de las enfermedades transmitidas por las garrapatas (Ixodidae) en los bovinos de Argelia, el autor estudió los caracteres estacionales de la actividad parasitaria en la región de Tiaret, Argelia. Entre enero y diciembre, de una muestra de 1240 bovinos examinados, 368 estaban infestados por garrapatas, con una tasa de infección global de alrededor de 30\%. La carga parasitaria mensual máxima fue de cinco garrapatas por animal. Entre las 3975 garrapatas recolectadas, se identificaron 13 especies, de las cuáles seis del género Hyalomma (66\%) y tres del género Rhipicephalus $(31 \%)$. Las especie dominante en cantidad fue $H$. lusitanicum $(20 \%)$, mientras que $H$. excavatum y $H$. marginatum marginatum presentaron las tasas de infestación de los bovinos más elevadas (13\%). Las tres especies monotrópicas, reconocidas como vectores de piroplasmosis bovinas en Argelia, representaron $37,4 \%$ de la población total de garrapatas. Entre estas, Rhipicephalus bursa representó 69\%, Hyalomma detritum 28\% y Boophilus annulatus 3\%. El clima semi árido de la región debe haber sido menos favorable para $B$. annulatus. La presencia de $R$. Bursa fue anual, mientras que la de $H$. detritum se limitó a alrededor cuatro meses, debido a una pausa invernal más prolongada de los pre imagos. La predominancia de $R$. bursa permitió pensar que las babesiosis deben ser más frecuentes en la región que la teilerosis (por Theileria annulata). La baja carga parasitaria observada y los numerosos casos de piroplasmosis señalados en los bovinos suponen la existencia de un problema de estabilidad enzoótica de estas enfermedades. Debido al riesgo potencial de infección durante ciertos periodos del año, principalmente para los bovinos importados, provocado por la predominancia de garrapatas adultas durante la estación caliente y de los pre imagos en la estación fría, debe administrarse un tratamiento acaricida preventivo entre mayo y octubre.

Palabras clave: Ganado bovino - Ixodidae - Metastigmata Garrapata - Piroplasmosis - Argelia. 